\title{
NUMERICAL MODELING OF ACCRETION COLUMN IN POLARS
}

\author{
C. Busschaert ${ }^{1,2}$, C. Michaut ${ }^{1}$, E. Falize ${ }^{2,1}$ and H.C. Nguyen ${ }^{3,1}$
}

\begin{abstract}
Using powerful lasers, we are now able to produce in laboratory relevant regimes of density, temperature and velocity to create a diagnosable exact scaled model of magnetic cataclysmic variables accretion column. We present here preliminary results of a numerical modeling of these astrophysical objects which will allow us to precise the experimental setup of future experiments.
\end{abstract}

\section{Introduction}

Magnetic cataclysmic variables (mCVs) are semi-detached binary system containing a magnetized white dwarf accreting materials from a companion through the inner Lagrange L1 point. Considering the specific case of polars, the magnetic field of the white dwarf is higher than $10^{3} \mathrm{~T}$, consequently the infalling material is directly led from the L1 point along the white dwarf magnetic field lines towards its magnetic pole(s) and there forms accretion column(s) (Wu 2000). Most of the radiation we receive from polars comes from a small area of the column near the surface of the white dwarf and spectroscopic clues indicate the presence of radiative shocks. In this high-energy environment the physical regime corresponds to the so-called case of optically thin radiative shock (Michaut et al. 2009).

Considering that size scales associated with the shocked region are of the order of $1000 \mathrm{~km}$, we cannot resolve this zone directly by observations. However, using adapted scaling laws, we can demonstrate that it is possible to reproduce with a powerful laser a diagnosable accretion column in laboratory (Falize et al. 2011a). Nowadays, reaching extreme states of density, temperature and velocity relevant for astrophysics has become feasible with modern high energy density facilities (Remington et al. 2006). In the POLAR Project, we take advantage of those possibilities to realize new similarity experiments of the formation and dynamics

\footnotetext{
${ }^{1}$ LUTH, Observatoire de Paris, CNRS, Université Paris-Diderot, 92190 Meudon, France

2 CEA-DAM-DIF, 91297 Arpajon, France

${ }^{3}$ Hanoi National University of Education, 136 Xuan Thuy, Cau Giay, Ha Noi, Vietnam
} 
of accretion shock in mCVs. The experimental concept is as follows: a flow of plasma is created through laser-matter interaction, the flow is then collimated by a tube and hits an obstacle to produce a diagnosable reverse shock. The tube plays the role of the magnetic field, which only collimates the infalling flow at the astrophysical scale, and the obstacle plays the role of the white dwarf surface. The first experiment, performed at LULI2000 laser facility of Ecole Polytechnique, allowed us to diagnose the reverse shock and to validate the experimental concept (Falize et al. 2012).

In this proceeding, we detail the numerical modeling at the astrophysical scale and present preliminary results of the accretion column base structure.

\section{Numerical modeling of the accretion column}

In order to explain observations of magnetic cataclysmic variables, the most common model is the following ( $\mathrm{Wu} 2000$ ). Material flows towards the white dwarf at supersonic velocity, $v \sim 5000 \mathrm{~km} / \mathrm{s}$, and hits its surface producing a reverse shock. Such velocity induces post-shock temperature of the order of $T \propto v^{2} \sim 10^{8} \mathrm{~K}$. Typical accretion rate for those object is $\dot{M} \sim 10^{-11} M_{\odot} /$ yr considering that the section of the column corresponds to a fraction $f \sim 10^{-4}$ of the white dwarf surface. The reverse shock propagates through the column until a position above the surface such that cooling processes in the shocked region allow material to have enough time to cool and slow down. The gravitational energy of the system is converted into thermal energy and then radiated away by different radiative processes such as bremsstrahlung or cyclotron emissions. Indeed, we assume that the plasma is optically thin since considering typical values in the column, it can be shown that the transverse optical depth, $\tau$, is approximatively equal to $8 \times 10^{-2}$ (Frank et al. 2002). This loss of energy implies a decrease of temperature from the shock front toward the surface of the white dwarf, accompanied by a densification and a decrease of velocity. Considering that the cooling timescales associated to those processes are smaller that the dynamic timescale of the system, those cooling processes entirely structure the base of the accretion column.

Analytical analysis of steady-state equations describing the column leads to determine the height of the accretion shocks as long as cooling loss can be expressed as a power law of density, $\rho$, pressure, $P$, and position (Falize et al. 2009). Applied to the case of polars considering only bremsstrahlung emission cooling, the cooling function is proportional to $\rho^{3 / 2} P^{1 / 2}$ and the shock height, $x_{s}$, is given by the relation:

$$
x_{s}=7.6 \times 10^{4} \mathrm{~m}\left[\frac{\dot{m}}{40 \mathrm{~kg} \mathrm{~m}^{-2} \mathrm{~s}^{-1}}\right]^{-1}\left[\frac{M_{W D}}{0.5 M_{\odot}}\right]^{3 / 2}\left[\frac{R_{W D}}{10^{7} \mathrm{~m}}\right]^{-3 / 2}
$$

compatible with the relation given by $\mathrm{Wu}$ et al. (1994), where $\dot{m}, M_{W D}, R_{W D}$ and $M_{\odot}$ are respectively the accretion rate, the mass of the white dwarf, its radius and the solar mass. 


\section{Preliminary results and perspectives}

HYDRO-COOL is a 2D hydrodynamical code developed at LUTH (Michaut et al. 2011). We use it to perform numerical simulations of the evolution of accretion column. HYDRO-COOL solves the Euler equations of hydrodynamics, where radiative losses are taken into account with a cooling function used as source term in the energy conservation equation. The cooling function only acts in the shocked region and when the temperature is higher than a critical value. For example, when bremsstrahlung process dominates the cooling, the critical temperature is $\sim 10^{6} \mathrm{~K}$. Below this value, the cooling process may be considered as inefficient. The algorithm is based on a MUSCL-Hancock scheme and the source term in the equations is taken into account by a Strang splitting method. The Riemann problem is solved either by a HLLE or HLLC method, both of them allowing to deal with high Mach number flows $(M>4)$.

At a first step, we validate the code through typical cases, using a plane-parallel geometry, to compare with for instance Chevalier \& Imamura (1982), Imamura et al. (1984), or more recently Mignone (2005). As an example of our preliminary results, we show the time evolution of the shock front position for a flow cooled by bremsstrahlung emission on a white dwarf of $1 M_{\odot}$, with a typical accretion rate of $\dot{M}=10^{-11} M_{\odot} /$ yr, using the steady-state profiles of density, velocity and energy as initial conditions (see Fig. 1). The shock front position oscillates along time with a time scale of the order of $0.2 \mathrm{~s}$, which corresponds to the cooling time scale. The steady-state predicted shock height is $x_{s} \sim 350 \mathrm{~km}$, and numerically $x_{s}$ oscillates around this theoretical value. The amplitude and frequency of oscillations are in agreement with previous studies. This oscillation induces a variation in

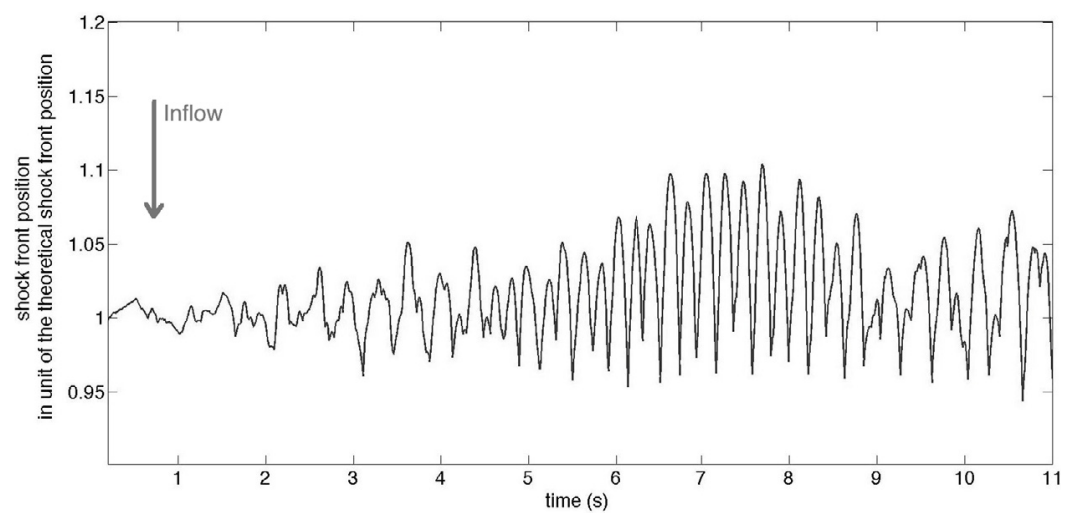

Fig. 1. Evolution of the position of the shock front with time. The surface of the white dwarf is at the origin of the $y$-axis. The oscillations are due to cooling instabilities which appear behind the shock.

luminosity, which could account for quasi-periodic oscillations (QPO) observed in some polars in optical wavelength, with a typical oscillation frequency around $1 \mathrm{~Hz}$ 
(e.g. see Larsson 1992). The oscillations comes from a cooling instability which develops behind the shock front, when the bremsstrahlung process cools the column (Langer et al. 1981). When the reverse shock is displaced toward (resp. away from) the white dwarf surface, the length of the shocked region decreases (resp. increases) and infalling material has less (resp. more) time to radiate and cool down. The excess (resp. shortage) of thermal energy inside the column leads to an excess (resp. lack) of pressure, which drives the shock upstream (resp. downstream). At late stage, the observed variation of the oscillation amplitude corresponds to the presence of several harmonics as expected from a perturbative analysis of the system (Chevalier \& Imamura 1982). In order to test this mechanism, we plan to go deeper into the simulation of this accretion process. Preliminary simulations seem to indicate that using a cylindrical geometry leads to qualitatively same results. Simulations are currently run in order to quantify more precisely the impact of using a cylindrical geometry and to explore the possibility of $2 \mathrm{D}$ simulations with spatially heterogeneous accretion or transverse perturbation for instance.

\section{Conclusion}

We present preliminary results of numerical simulation at the astrophysical scale of accretion column in cataclysmic variables. These numerical simulations will soon be improved in order to take into account several cooling functions and add physical components, which may become important depending on the accretion regime. The $2 \mathrm{D}$ geometry will also be studied in order to evaluate more accurately the impact onto the system dynamics.

\section{References}

Chevalier, R.A., \& Imamura, J.N., 1982, ApJ, 261, 543

Falize, E., Michaut, C., Bouquet S., et al., 2009, Ap\&SS, 322, 71

Falize, E., Loupias, B., Ravasio A., et al., 2011a, Ap\&SS, 336, 81

Falize, E., Michaut, C., \& Bouquet S., 2011b, ApJ, 730, 96

Falize, E., Ravasio, A., Loupias, B., et al., 2012, HEDP, 8, 1

Frank, J., King, A., \& Raine, D.J., 2002, in Accretion power in astrophysics, third edition (Cambridge University Press)

Imamura, J.N., Wolff, M.T., \& Durisen, R.H., 1984, ApJ, 276, 667

Langer, S.H., Chanmugam, G., \& Shaviv, G., 1981, ApJ, 245, L23

Larsson, S., 1992, A\&A, 265, 133

Michaut, C., Falize, E., Cavet, C., et al., 2009, Ap\&SS, 322, 77

Michaut, C., Nguyen, H.C., \& di Menza, L., 2011, Ap\&SS, 336, 175

Mignone, A., 2005, ApJ, 626, 373

Remington, B.A., Drake, R.P., \& Ryutov, D.D., 2006, Rev. Mod. Phys., 78, 755

Wu, K., Chanmugam, G., \& Shaviv, G., 1994, ApJ, 426, 664

Wu, K., 2000, Space Sci. Rev., 93, 611 